\title{
Automated Computer-Based Facility for Measurement of Near-Field Structure of Microwave Radiators and Scatterers
}

Mishra, Shantnu R.;; Pavlasek, Tomas J. F.;; Muresan, Letitia V.

Published in:

I E E E Transactions on Instrumentation and Measurement

Link to article, DOI:

10.1109/TIM.1980.4314979

Publication date:

1980

Document Version

Publisher's PDF, also known as Version of record

Link back to DTU Orbit

Citation (APA):

Mishra, S. R. ., Pavlasek, T. J. F. ., \& Muresan, L. V. (1980). Automated Computer-Based Facility for Measurement of Near-Field Structure of Microwave Radiators and Scatterers. I E E E Transactions on Instrumentation and Measurement, 29(4), 455-461. https://doi.org/10.1109/TIM.1980.4314979

\section{General rights}

Copyright and moral rights for the publications made accessible in the public portal are retained by the authors and/or other copyright owners and it is a condition of accessing publications that users recognise and abide by the legal requirements associated with these rights.

- Users may download and print one copy of any publication from the public portal for the purpose of private study or research.

- You may not further distribute the material or use it for any profit-making activity or commercial gain

- You may freely distribute the URL identifying the publication in the public portal 


\title{
Automated Computer-Based Facility for Measurement of Near-Field Structure of Microwave Radiators and Scatterers
}

\author{
SHANTNU R. MISHRA, MEMBER, IEEE, TOMAS J. F. PAVLASEK, SENIOR MEMBER, IEEE, AND \\ LETITIA V. MURESAN, MEMBER, IEEE
}

\begin{abstract}
An automatic facility for measuring the three-dimensional structure of the near fields of microwave radiators and scatterers is described. The amplitude and phase for different polarization components can be recorded in analog and digital form using a microprocessor-based system. The stored data are transferred to a large high-speed computer for bulk processing and for the production of isophot and equiphase contour maps or profiles. The performance of the system is demonstrated through results for a single conical horn, for interacting rectangular horns, for multiple cylindrical scatterers, and for the fields inside an absorber lined chamber.
\end{abstract}

\section{INTRODUCTION AND SYSTEM DESCRIPTION}

QOME CURRENT activities in the analysis and synthesis $D$ of radiating systems or scatterers have stimulated interest in the investigation of their near-field structure. For example, the relationship between current distribution and the near field is useful in understanding input impedance behavior [1] while for complex structures the near field can give insight to interaction and coupling between radiators and nearby passive objects [2]. Similarly, the immediate near field of scatterers can provide insight into diffraction and scattering mechanisms. Several practical motivations direct attention to near-field structure, in particular the emerging techniques of near-tofar-field transformation [3], and also the analysis of radiator interaction as encountered in electromagnetic compatibility problems [4].

In designing experimental equipment for near-field measurements, the quantities whose measurement is indicated are the amplitude and the phase of the various field components and, because of the field complexity, the three-dimensional structure may need to be determined. Apparatus for such measurements are characterized by the large number of data points which must be obtained, thus requiring substantial automation of the measurement process. The recording of data and their presentation in a meaningful form must rely on computerized techniques for data acquisition and its processing into suitable graphical form. An effective form is that of iso-

Manuscript received June 28, 1980; revised September 10, 1980. S. R. Mishra and T. J. F. Pavlasek are with the Department of Electrical Engineering, McGill University, Montreal, P.Q. H3A 2A7, Canada.

L. V. Muresan is with the Electromagnetics Institute, Technical University Denmark, Lyngby, Denmark. phot and equiphase maps of the field in selected sectional planes which, in ensemble, display the three-dimensional structure. Experience indicates that single maps for simple radiators (e.g., a single horn) require the acquisition and management of measurements at about 10000 positions while more complex structures require data points of the order of 100000 for each sectional plane.

This paper describes such equipment built for operation at $23.5 \mathrm{GHz}$ or, optionally at other frequencies in the microwave region from $0.6 \mathrm{GHz}$ and upwards. The facility consists of automated measuring equipment which determines the amplitude and phase of the near field of a radiating antenna or a scatterer. The measurements are made throughout a volume of space surrounding the object, are carried out in an anechoic room, and the resulting digitized data are processed into maps or other graphical form using a large high-speed computer interfaced with the facility's dedicated microprocessor-based computer. The system is described diagrammatically in Fig. 1. The equipment can also be used for the determination of microwave two-port parameters when arranged in a closedcircuit form.

\section{Method of MEASUREMENT}

The measurement determines the field's relative amplitude and phase. A sensing probe (small antenna) is placed in the field and is located by a triaxial optical bench system which allows positioning of the probe anywhere in a volume of the space enclosed by the anechoic chamber. The scanning is in cylindrical coordinates, the $z$ axis corresponding to the optical axis of the measured radiating system, as illustrated in [5]. In the $K$-band $(23.5-\mathrm{GHz})$ form of the equipment the positioning system scans a cylindrical volume approximately $100 \lambda$ in diameter and about $150 \lambda$ along the axis. The probe is moved along radial straight line scans and positioning accuracy is better than $\lambda / 12$.

The probes used are usually in the form of tuned or small dipoles although other probes may be used as well.

The field amplitude and phase are measured using a homodyne system [5]. In this system, a CW carrier source is separated into two paths, one of which is suppressed carrier double-sideband modulated and the other is used as a reference 


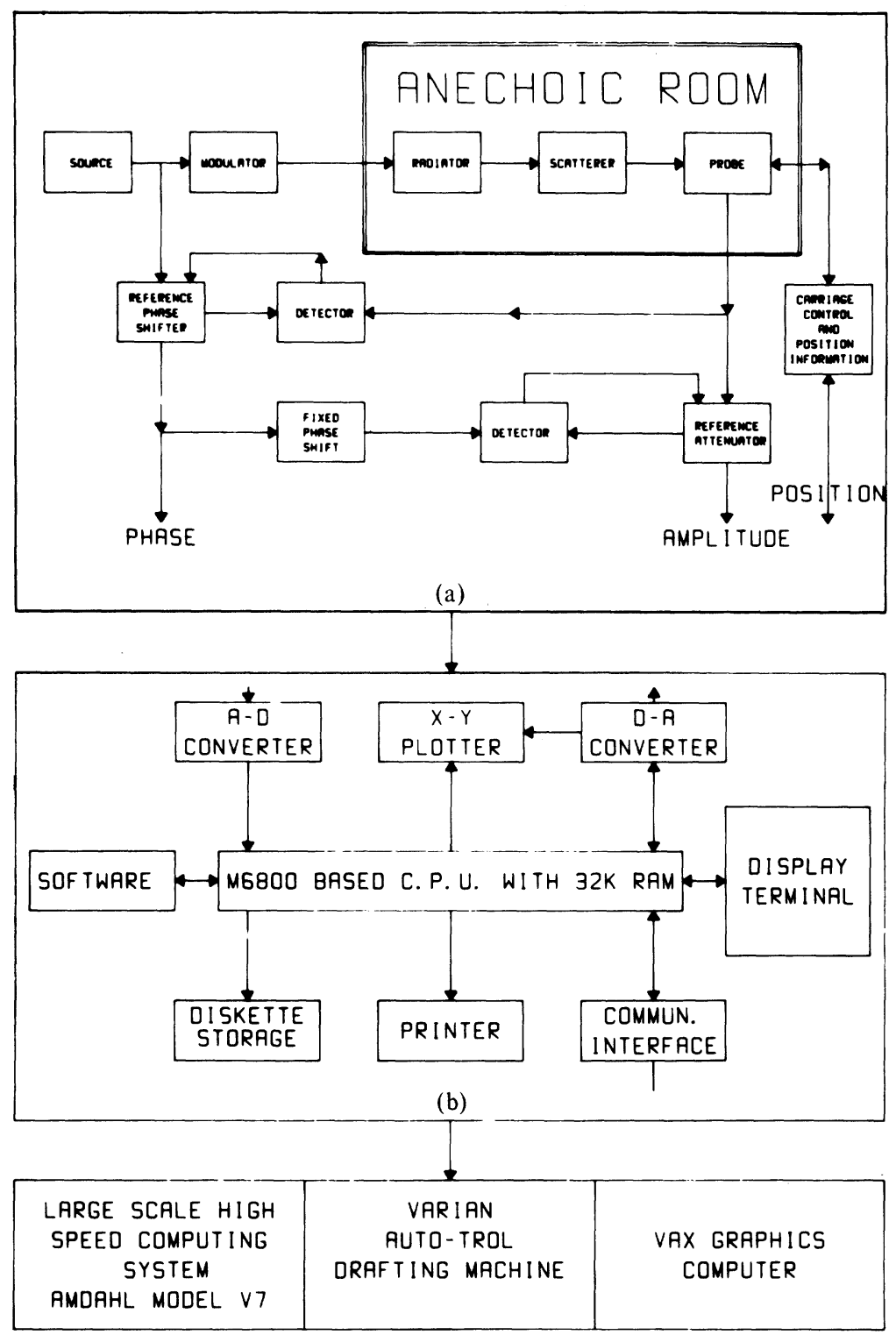

(c)

Fig. 1. Block diagram. (a) Measuring. (b) Data acquisition. (c) Map plotting system.

carrier. The balanced-modulated signal is used to energize the radiating antenna under test or to illuminate a scatterer under study.

The advantages of this system are that the measurement of amplitude and phase are independent of each other. Secondly, both amplitude and phase are determined by substitution in terms of a reference precision attenuator and a precision rotary phase shifter. The amplitude accuracy is better than $1 \mathrm{~dB}$ and the phase accuracy is approximately $5^{\circ}$ in the dynamic, automatic mode of operation. The potential dynamic range is of the order of $120 \mathrm{~dB}$ but is limited by the range of the substitution attenuator. The phase range is essentially unlimited. Improved accuracy can be obtained by resorting to a manually controlled mode of operation when small local regions of a field merit particular attention.

In comparison with available commercial systems using open-ended-type measurements, this system, in addition to the wide dynamic range and an essentially unlimited phase range, has substantial low noise advantages. Furthermore, the measurement is in terms of substitution elements for amplitude and phase, which can be directly calibrated against reference standards if necessary and the calibrations incorporated into the digital data system. When operating under manual control the accuracy can be improved considerably. The accuracy is then $0.2 \mathrm{~dB}$ for amplitude and $0.5^{\circ}$ in the case of phase but this is achieved at the cost of reducing the speed of measurement.

For operation at lower frequencies the homodyne system may be replaced by a Network Analyzer (hp $8410 \mathrm{~A}$ ) or a Vector Voltmeter (hp $8405 \mathrm{~A}$ ). In this form some reduction in accuracy, operating range and data acquisition convenience may be expected. 


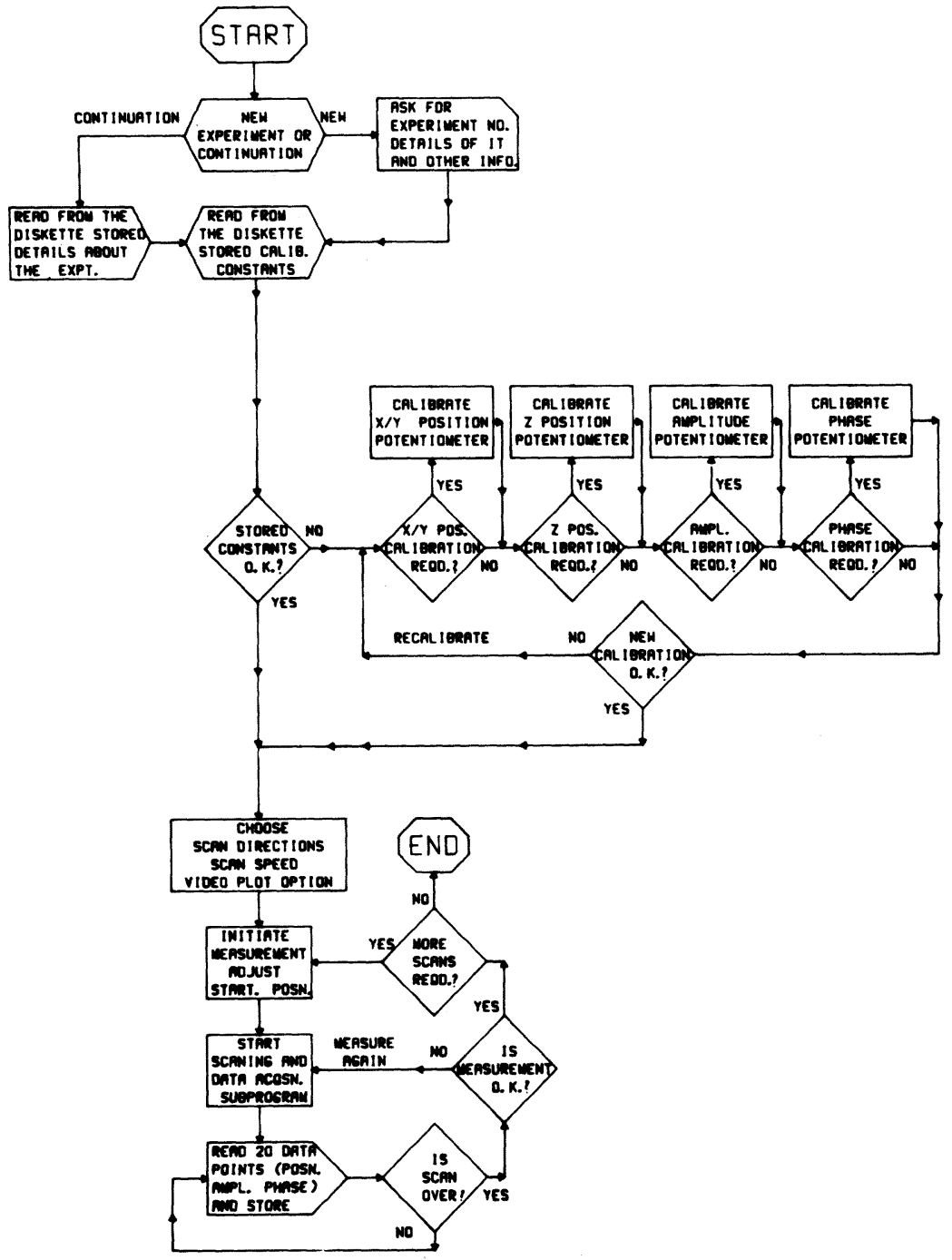

Fig. 2. Measurement control and data acquisition software flow chart.

\section{CONTROL OF EXPERIMENT, DATA ACQUISITION, AND PROCESSING}

The control, the acquisition of data and its processing are structured on a microprocessor-based system (Motorola 6800). As shown in Fig. 1, this system includes analog/digital conversion, floppy diskette storage, digital/analog conversion for process control, a video and hard copy terminal, and a communication interface to a large high-speed computer.

This system allows: for the control of the experiment, for the digital encoding of the measured data, for the bulk storage of the data, and for its subsequent transfer to a high-speed computer for processing into a form suitable for graphical display such as maps. If desired, the measured data can be displayed and recorded in analog graphical form in real time for monitoring purposes.

The measured data are basically available in terms of mechanical shaft positions of the reference attenuator, the phase shifter, and the probe position optical bench lead screws. These are encoded into digital form for storage and processing or converted to electrical analog form for real-time monitoring and display.

The software which has been developed (Fig. 2) includes subroutines: to digitize the amplitude, phase, and position information; to store the data in organized disk (floppy diskette) files already suitably scaled for future processing; to monitor the progress of measurements, including initial calibration procedures; to maintain directories and index files which provide direct access to any specific data scan or data point; to display a plot of data being measured in real time on a video terminal. Provision is made for controlling the probe position through the microprocessor thus giving opportunity for programmed positioning operation of the system, for example, enabling the probe to follow amplitude or phase contours.

In order to process the data in bulk for the production of maps etc., software has been developed to interface the microprocessor system with an AMDAHL large-scale high-speed computer through a serial line using available interactive computing facilities (MUSIC). The data are transferred to on-line disk files of the AMDAHL computer and then various 


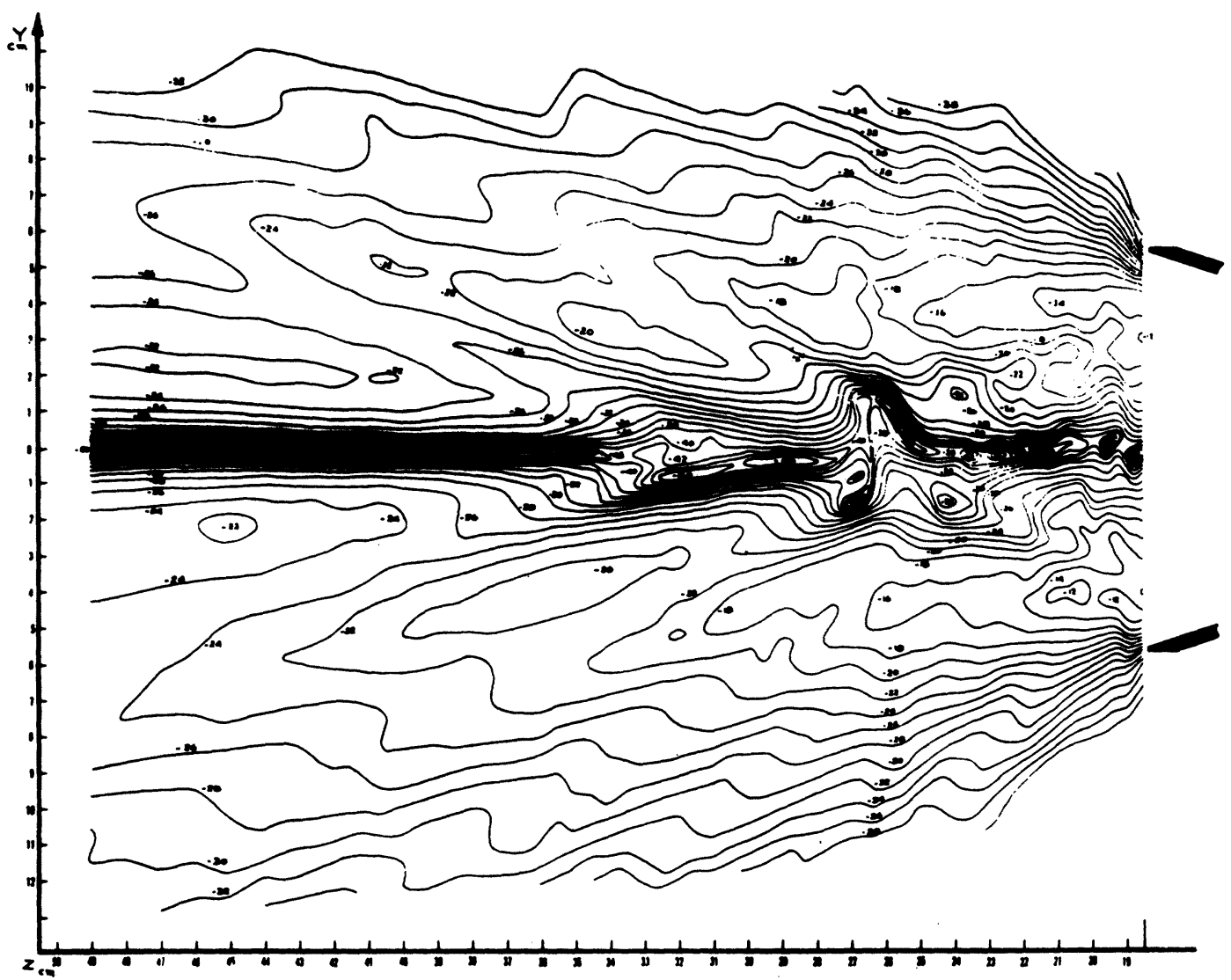

(a)

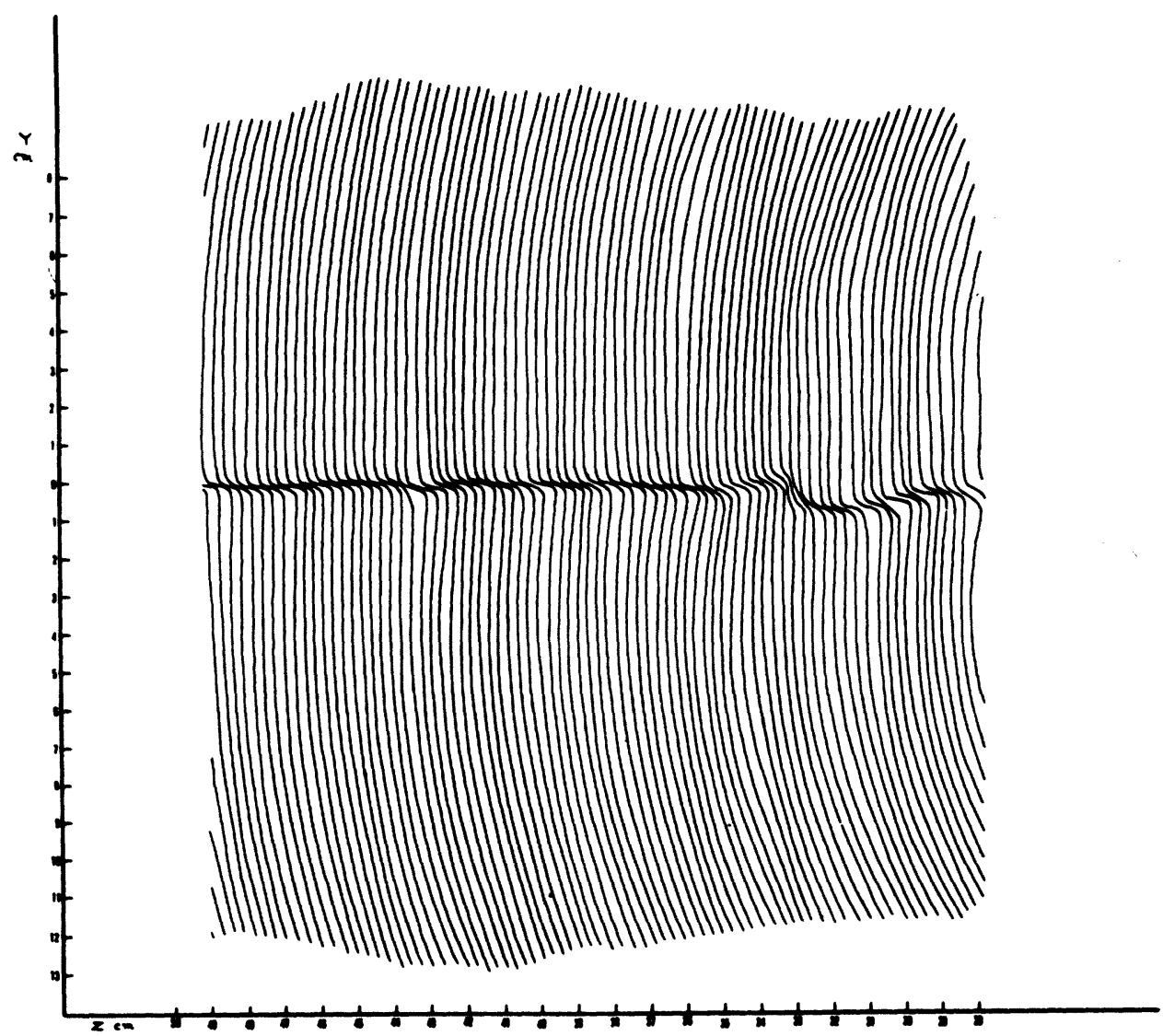

(b)

Fig. 3. Measured (a) isophot and (b) equiphase maps of the cross polarized $E$ field in the mouth of a conical horn excited in the $T E_{11}$ mode. 

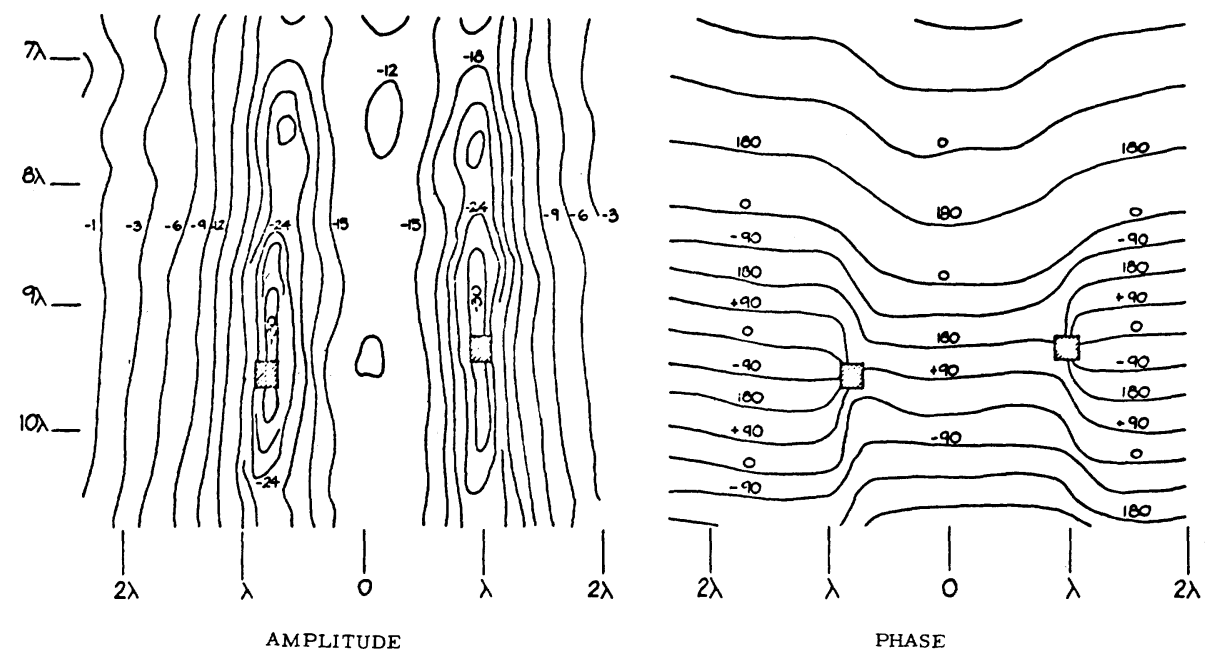

Fig. 4. Details of amplitude and phase field structure in region of nulls in shadow of two scattering cylinders.

interpolation, plotting, and other data processing routines are used to produce contour maps or section profiles and other forms of graphical display. This form of mapping preserves the quantitative nature of the information, which makes it useful for interpretation and for comparison with similar maps produced by theoretical numerical computation.

In addition there is provision for similar data transfer and processing on a VAX Graphics computer for the production of qualitative graphical display in pseudocolor.

\section{System Performance AND Results}

The system has been used to measure the fields of a wide range of different cases. These have included simple radiators such as horns, diffracting and focusing apertures such as lenses, scattering by multiple objects of different materials and shapes. A selection of cases is presented here to illustrate the capabilities of the system (Figs. 3, 4, 5,6) and certain aspects of its performance.

Time is an essential parameter in evaluating the system. Manual or semiautomatic apparatus used in early versions required several days to measure a relatively simple map of a horn whose maps are shown in Fig. 3. Depending on the complexity, the subsequent manual map drawing required many days up to several weeks. The present system can measure and acquire the data for a complex situation such as Fig. 6 (anechoic room) in one working day. Such a map is produced by making approximately one hundred line scans of the field, each scan lasting from 5 to $10 \mathrm{~min}$, depending on the complexity of the field structure. Approximately "one data point" is recorded per second and the scanning speed is controlled in a manner to obtain sufficient detail in a rapidly changing field structure. Each "data point" includes the amplitude, the phase, and the three position coordinates in space. The speed limitation at the present time is not in the data acquisition process but in the measurement which involves the mechanical amplitude and phase substitution elements along with the electromechanical null-seeking techniques [5]. A system involving digitally controlled microwave attenuators and phase shifters is under development to overcome this speed limitation. The measurement speed would then be limited by the probe transport.

Although the map production requires only about one to two service units of the operating system and about an hour of CALCOMP off-line plotting time, the outputting of a map usually entails a second working day because of the usual operational scheduling of a large computer. The plotting time could be made shorter readily, but at this stage of development the production of maps is limited by the measurement time.

An important aspect of this system is the measurement of phase. In many cases the structure of a field may contain low-level regions or nulls which are not distinguishable from each other by amplitude measurements alone, due to noise limitations. Similarly single point or single scan profiles do not provide sufficient information even in terms of rapid phase change to allow discrimination between nulls and low minima. Such information however, is contained in the two-dimensional maps of equiphase contours which are characterized by a multivalued phase structure at the null locations [6]-[8]. The detailed examination of such regions requires the phase information to diagnose the presence of an absolute null. Structures illustrating this behavior are shown in Figs. 4 and 5 . The null locations in some cases may characterize the behavior of scatterers in a unique one-to-one correspondence between the scatterer parameters and the nulls [9]. Fig. 6 illustrates the system capability of producing maps over a relatively extended region. This shows the field structure present within an absorber lined chamber illuminated by a dipole radiator operating at a frequency about one order of magnitude below the normal rated frequency of the absorber material. This indicates the degraded behavior of an anechoic room operated below its lowest rated frequency.

Finally the system can be used not only for field measurements but also in a "closed circuit" configuration. In this form it is useful for the determination of microwave two-port parameters as, for example, the determination of liquid crystal characteristics as a function of temperature and magnetic field at the phase transition point [10]. 

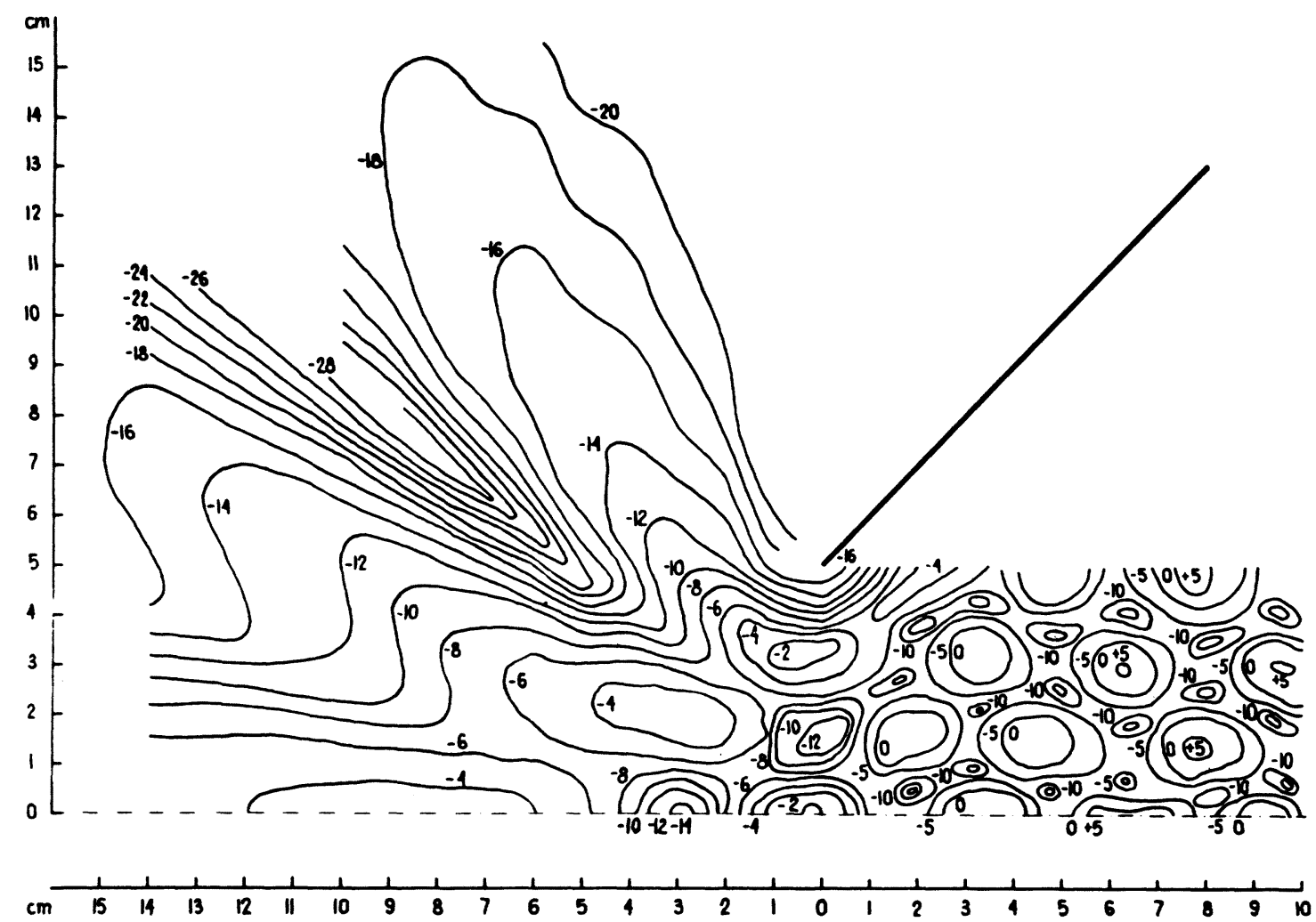

(a)
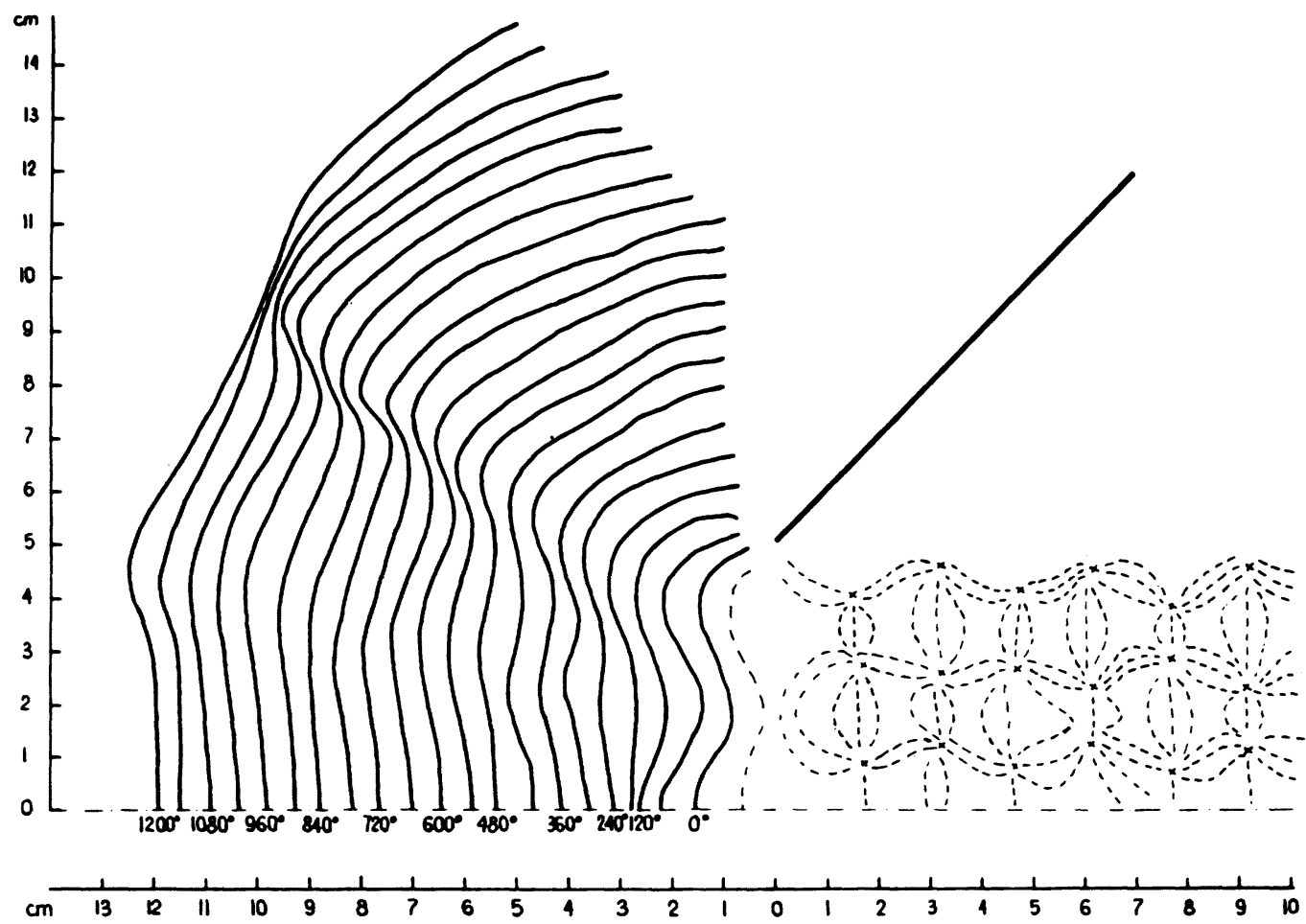

(b)

Fig. 5. Isophot and equiphase map in and near slit aperture formed by two inclined semiinfinite conducting planes. (a) Isophot map. (b) Equiphase contour map (contours are shown at $90^{\circ}$ intervals). 


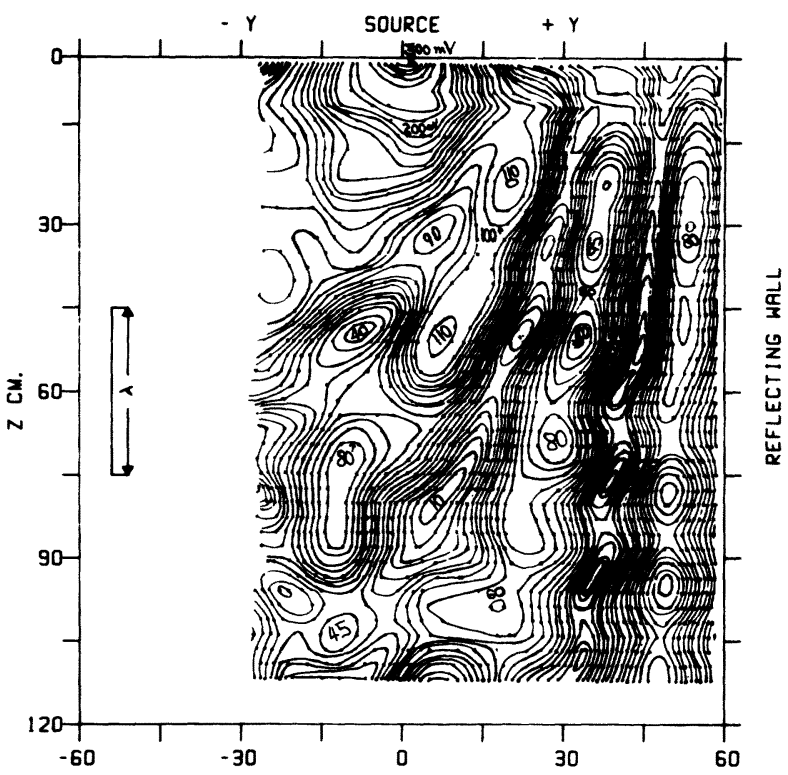

(a)

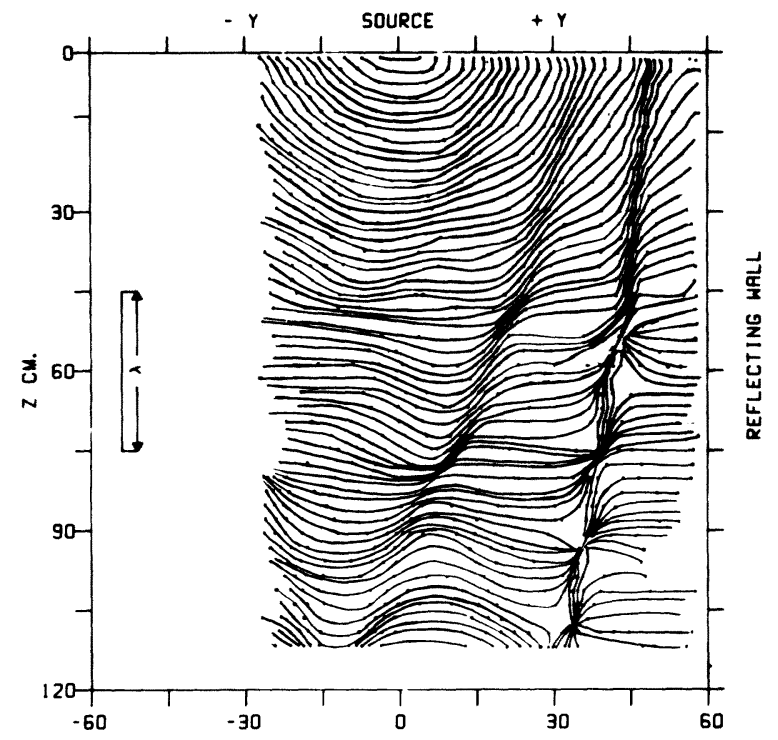

(b)

Fig. 6. Field structure in small absorber lined chamber (ALC) operated at $1 \mathrm{GHz}$ with reflecting side wall using absorber designed for anechoic chamber use at $10 \mathrm{GHz}$. (a) Isophot map. The relative amplitude of principal peaks is indicated. (Contours are at $5-\mathrm{mV}$ intervals except near the source where $10-\mathrm{mV}$ intervals are used.) (b) Equiphase contour map (contours are shown at $30^{\circ}$ intervals).

\section{REFERENCES}

[1] R. W. P. King, Theory of Linear Antennas. Cambridge, MA: Harvard Univ. Press, 1956, ch. II.

[2] F. L. Cain et al., "Prediction of near field antenna coupling in presence of obstacles," 1972, in IEEE Int. Symp. E.M.C., Arlington Heights, IL.

[3] R. C. Johnson et al., "Determination of far field antenna patterns from near field measurements," Proc. IEEE, vol. 61, no. 12, pp. 1668-1694, Dec. 1973.

[4] C. E. Ryan and F. L. Cain, "Techniques for estimating E.M. effectiveness of antennas in the presence of near field obstacles," in 1976 IEEE Symp. E.M.C., Washington, DC, pp. 317-322, July 1976.

[5] B. Howarth and T. Pavlasek, "Analysis of automatic homodyne method:
Amplitude and phase measurements," IEEE Trans. Microwave Theory Tech., vol. MTT-20, pp. 623-626, Sept. 1972.

[6] W. Braunbek and G. Laukien, Optik, 9, p. 174, 1952.

[7] L. Lee and G. W. Farnell, "Phase singularities for microwave lenses with aberrations," J. Opt. Soc. Amer., vol. 51. no. 3, pp. 273-277, 1961.

[8] Y. F. Lum and T. J. F. Pavlasek, "The influence of aberrations and aperture inclinations on the phase and intensity structure in the image region of a lens," IEEE Trans. Antennas Propagat., vol. AP-12, pp. 717-727, Nov. 1964.

[9] B. Howarth et al., "A graphical representation for interpreting scalar wave multiple-scattering phenomena," J. Comput. Phys., vol. 15, no. 2, pp. 266-285, June 1974.

[10] G. Nimtz et al., "Even-odd effect and chain configuration in P-Alkoxybenzal-p'-Ethoxyaniline," Solid State Communications, vol. 29, pp. 287-290, 1979. 